\title{
Should consultant psychiatrists be trained in psychotherapy?
}

\author{
Nick Temple
}

Psychosocial aspects of treatment have become increasingly important with the changes in psychiatric practice away from the hospital to the community. Effective psychosocial interventions and high staff morale are likely to be the key to the success of a community-based mental health service. There is a growing expectation that all psychiatrists should have a basic knowledge of therapeutic approaches in order to take a leading role in the multi-disciplinary team. They should be able to assess patients for a range of psychological and psychotherapeutic treatments. This role is vital as the matching of the patient's needs with the appropriate treatment is the key to a successful outcome.

There is continuing concern from the Government about the perceived failure of community mental health care (Dobson, 1998). The high turnover of staff in many services has contributed to this and, together with worrying levels of sickness, indicates a service which is under pressure. This emphasises the importance of finding effective clinical leadership for the multi-disciplinary team, who need to experience their own efforts as resulting in improved patient care.

The NHS review of psychotherapy services (NHS Executive, 1996) recommended that all mental health practitioners should have a basic training in psychotherapy and psychological treatments, to enable them to use these principles in their everyday work with patients. In addition, many mental health staff need training which will enable them to provide psychotherapeutic treatment in one of the main modalities. At present, the majority of practising consultant psychiatrists do not measure up, even to the minimum requirements of training proposed by the review.

\section{Types of psychotherapy}

Cawley (1973) described four levels of psychotherapy and this has been broadly adopted by the NHS review of psychotherapy services. Level 1 refers to psychotherapeutic technique and knowledge which should be part of the general skill of all mental health practitioners. For example, the use of the concept of transference as a phenomenon in all therapeutic interactions provides an essential insight into the management of patients in all mental health settings. Level 2 defines specific psychotherapeutic treatments such as brief psychotherapy or cognitive-behavioural therapy (CBT), but practised at an eclectic non-specialist level. Level 3 refers to specialist psychotherapy requiring a fullscale training in one of the main therapeutic approaches. Cawley's Level 4 represents behavioural psychotherapy. The review has adopted Cawley's Levels 1, 2 and 3 as Type A, B and C. Level 4 -behavioural psychotherapy - is incorporated as one of the modalities of psychotherapy practice in Type C. It recommends that all consultant psychiatrists and other mental health professionals should be familiar with Type $A$ and incorporate it in their clinical management of patients and in their understanding of patients' psychopathology. There is a strong argument that many psychiatrists should also be able to practise at least one form of Type B psychotherapy to provide an effective input to their practice and to supervise others in this work. This is normal in many other countries where psychotherapy is a standard part of psychiatric training, e.g. USA, Australia, New Zealand and Finland. Type $C$ is specialist psychotherapy in one of the main

Nick Temple is Consultant Psychotherapist in the Adult Department at Tavistock Clinic (120 Belsize Lane, London NW3 5BA) and has been Chairman of the Tavistock Clinic since 1993. He has extensive experience of teaching psychotherapy. He was also Chairman of the Psychotherapy Section, Royal College of Psychiatrists 1990-1994 and is currently a member of the College Court of Electors. 
modalities. The review recommends that a comprehensive specialist service is provided as a part of every district mental health service and that this is closely integrated to the community mental health service.

The NHS policy on clinical governance (NHS Executive, 1998) is designed to ensure a high standard of clinical practice in all NHS professions through CPD, clinical effectiveness, professional review and audit. The continuing crisis in the mental health service will lead to inevitable questions about the level of training available to staff and the leadership needed to deliver an adequate service. Increased numbers of complaints from patients under the Patient's Charter also focus attention on the quality of training needed to provide an effective and safe service.

A vital question concerns the future leadership role of the consultant psychiatrist in the community mental health service. Is the consultant psychiatrist equipped to lead a generic mental health team? Or will the psychiatrist's function be a more narrowly specialist one - that of the diagnostic assessment and drug treatment of seriously mentally ill patients? It seems that the latter function does not require the consultant psychiatrist to have a training in psychosocial and therapeutic approaches. It also restricts their leadership function in the community mental health service. Other mental health professionals are ready to take over the organisation and delivery of psychological treatments and the leadership of the mental health team. If this happens, the role of the psychiatrist will be diminished and, as the general psychiatrist still has the broadest training in the diverse aspects affecting mental health, this will ultimately be to the detriment of the overall service to patients.

\section{Psychotherapy within general professional training}

In 1993, the General Psychiatry Section and the Psychotherapy Section together produced the Guidelines for Psychotherapy Training as part of General Professional Psychiatric Training (Royal College of Psychiatrists, 1993). A revised version of these guidelines is being produced, to be published in 1999. These were endorsed by the Court of Electors of the College and became mandatory for all SHO training schemes. The guidelines state that psychotherapeutic skills and knowledge are a core part of psychiatric practice and not just the preserve of specialist psychotherapists - just as intuitive psychological skills have always been the hallmark of a good doctor. The guidelines state that 'all psychiatrists, whatever the branch in which they ultimately specialise, require to develop interview and communication skills. They need to be able to handle emotionally stressful situations with sensitivity and awareness'. In order to achieve these aims, the guidelines specify that each psychiatrist needs to have a training in psychotherapy and human relations skills as part of their general professional education. It is not proposed that each psychiatrist needs to have a specialist level of expertise in psychotherapy, but rather training should aim to provide a working knowledge of therapeutic methods and an ability to assess a patient's suitability for a range of psychological treatment approaches. An additional aim of training is to provide the consultant psychiatrist with a personal awareness of their own interaction with the patient and to be able to recognise subtle aspects of the doctor-patient relationship. This will include negative feelings in both patient and doctor which are often avoided. Work discussion groups are to be provided in each training scheme to support and facilitate this kind of learning from experience.

The guidelines recommend that training in interviewing techniques is provided in the first year and that trainees take part in a work discussion group to discuss their general clinical work. The focus is on the doctor-patient interaction, where clinical and management problems can provide excellent training material. Experience of personal therapy can be a valuable adjunct to this experience.

Box 1. Psychotherapy review (NHS Executive, 1996)

Types of psychotherapy

Type A Psychological treatment as an integral component of mental health care, offered by mental health and primary care staff alongside other types of care

Type B Eclectic psychological therapy and counselling. Complete interventions informed by more than one theoretical framework

Type C Formal psychotherapies. Specialist practice within particular models e.g. psychoanalytic, cognitivebehavioural, systemic 
In the second year, trainees gain experience of supervised psychotherapy of an intensive case in dynamic psychotherapy and in CBT. They also have the opportunity to treat a group and a marital couple.

An important part of psychotherapy training is supervised work with an individual patient over an extended period. The supervisor encourages the trainee to study the fine detail of the interaction with the patient through observation and written records of the session. The practical considerations of setting boundaries of time and place are emphasised in supervision, and it is possible to observe and learn from the patient's reactions to changes made in these aspects of the treatment structure.

The close consideration of the work in progress encourages reflective practice as opposed to a reactive response. This experience is often greatly valued by trainees as one of the most helpful aspects of their training.

Supervision in small groups allows trainees to hear about the progress of others' work and to learn about a wider range of clinical situations. This detailed observational training is very relevant to developing the general psychiatrist's skills in understanding and managing patients, regardless of whether they are suitable for psychotherapy. This process enhances the clinician's resilience and depth of knowledge. Supervisory work has an important bearing on the clinical team's capacity to manage the strain of caring for ill patients by providing a strong framework which enables the staff to make emotional sense of what is going on in their patients' and their own reactions.

The College guidelines adopt the view that a knowledge of psychotherapy makes a central contribution to the basic communication skills and human understanding of the psychiatrist. Although these aspects of the doctor's function are likely to be well developed in those who choose psychiatry as a profession, natural ability needs to be developed by education and training. Training will enhance the doctor's capacity to observe and identify the subtle aspects of the doctor-patient relationship which are complex and may not at first be obvious to a scientifically-trained clinician. The hidden aspects of the relationship need attention as they will affect the patient's feeling towards the doctor and may distort the doctor's attitude to the patient. The ability to identify the nature of this interaction is an essential part of the clinician's diagnostic skills the psychiatrist needs to be able to diagnose patterns of interaction and relationships if he is to be able to contribute to a team's capacity to understand and fully care for a disturbed patient.

This approach to understanding the doctorpatient relationship has been influential in general practice and was originated by Balint (1957) who used work discussion groups as a way of improving the GP's effectiveness in the psychological aspects of their medical practice. In recent years, work discussion groups have become a standard part of GP training, where knowledge of the psychological and emotional aspects of the doctor-patient relationship is regarded as a basic part of their medical skills. Training in a Balint Group enhances the psychological resilience of GPs. It has proved to be helpful in improving the GP's capacity to maintain a sound psychological state in themselves, and to deal with difficult emotional situations which arise in their work with patients (Balint \& Balint, 1961).

\section{Problems of providing psychotherapy training for general psychiatrists}

A survey of training schemes for SHOs in psychiatry carried out on behalf of the Court of Electors (Gibb \& Temple, 1998) has shown that, despite the existence of the guidelines, 5 years after their publication, only about half the training schemes are providing adequate training in psychotherapy. Where provided, it is often not taken up by SHOs: at present, it is not mandatory for trainees to take up available training.

The slow progress in providing mandatory psychotherapy training to the agreed standards seems to indicate that, despite College policy, there are problems in giving this training sufficient priority. Perhaps psychotherapy training, focusing on an understanding of the patient's emotional life, is thought to weaken scientific psychiatric practice. It will be difficult for the trainee to engage with these issues unless there is support and encouragement from trainers.

At present, there may be reluctance on both sides to take on the complex and potentially difficult area

Box 2. Components of psychotherapy training in RCPsych guidelines in general professional training

Year 1 Training in interview techniques Work discussion group to explore psychotherapeutic principles in general psychiatric practice

Year 2 Supervised cases in

(a) psychodynamic

(b) cognitive-behavioural therapy Supervised group psychotherapy and marital therapy 
of the psychiatrist's own emotional responses and how this can affect interaction with a patient. It can be helpful for trainees to have their own personal therapy to recognise how their own emotional problems may become problems in the work setting. Many training schemes do not have sufficient sessions for the consultant psychotherapist to provide adequate supervision, or do not have a consultant psychotherapist available to contribute to the training at all.

\section{Interviewing skills and interaction with the patient}

The psychiatrist's skill in interviewing is a central aspect of both the conduct of an interview and the management of the relationship with the patient. Close attention and observation of the interactions between the patient and doctor will produce valuable insight into the patient's inner mental life and its relationship to the psychiatric disturbance.

The psychiatrist can improve his contact with the patient by observing and managing the patient's experience of the interview. Some practitioners control the interview so as to avoid the patient feeling anxious or distressed and to ensure that, at least on the surface, the patient feels the psychiatrist to be a benign supportive figure. This management of the relationship may be necessary in dealing with a paranoid or very anxious patient, but it may be detrimental to a patient who needs help to face anxieties and problems. It could be unhelpful to put the psychiatrist in a position where he is seen as being all powerful and able to put things right. Psychotherapeutic training will enhance the psychiatrist's capacity to detect the patient's wish to invest the doctor with ideal characteristics, and will help to avoid the tendency for the relationship to become one based on reassurance and avoidance of anxiety and hostility. It can also offer the psychiatrist insight into his own motivation and help him to identify ways in which he may wish the patient to think well of him and is anxious about a more negative interaction.

\section{Dependency and supportive counselling}

There has been much discussion about the place of supportive counselling in psychiatric practice. It may be necessary to strike up a benign relationship with the patient but, as has been pointed out by Feldman (1993), problems can arise when reassurance becomes a misleading basis of support for the patient's functioning. On the other hand, the support given by regular contact with a psychiatrist can be of the greatest benefit in the long-term management of very disturbed patients. For example, some patients with severe antisocial personality disorders are contained and supported by long-term regular appointments with a forensic psychiatrist, which will help to prevent the most disturbed aspects of the patient being acted out.

The consultant psychiatrist will find useful and effective an understanding of the nature of supportive relationships and an awareness of the significance of dependency wishes towards the idealised figure of the doctor. Sometimes the patient's dependency is seen as damaging and opposed to treatment (Balint, 1968), yet a damaged or disturbed patient needs access to reliable emotional support and needs to be appropriately dependent. The balance of this is crucial to the patient's response to treatment. In certain settings such as day hospitals, psychiatric patients can become dependent and use the treatment setting to avoid responsibility for their own lives, but they can be helped by treatment aimed at challenging dependency (Temple et al, 1997). The psychiatrist needs to be able to assess these situations and judge when dependence and psychological regression may be vital to the patient's well-being, and at other times when dependence may be used in a negative and harmful way (Balint, 1968; Stewart, 1989).

\section{Therapeutic community methods applied in the ward}

One important aim of the mental health team is to run a well-functioning ward which supports the patients and minimises disruption derived from their disturbance. In addition, it will need to ensure that day hospitals and residential hostels are supportive and do not encourage disruptive or regressive behaviour in the patients. There is much to be learnt from the therapeutic community approach in achieving this aim: here, interactions on the ward are used to further therapeutic work and groups are set up to facilitate the process. For example, daily ward groups can have an important integrating effect and similar groups can be set up in hostels and day units.

Therapeutic communities have been shown to be effective in the treatment of personality disorders - 
publication of results from the Henderson Hospital (Norton \& Hinshelwood, 1996) gave rise to the recommendation of more widespread adaptation of this approach because of its effectiveness. It uses the institutional setting as a means of bringing about psychosocial change. Every psychiatric ward should have an element of therapeutic input to its organisation to potentiate the effectiveness of other treatment approaches and to contain disturbed interactions between patients, and between staff and patients. This is often not adequately addressed in the training of staff who are running wards, so that they are not equipped to make use of well established principles to run the ward effectively. If the psychiatrist who is responsible for a ward or community service or hostel is not aware of the need to manage a ward to enhance its therapeutic possibilities, it is likely to be in difficulties owing to a weakening of its capacity to act as a container for emotional disturbance.

\section{Leading a mental health team}

The consultant psychiatrist needs to be equipped with an adequate level of knowledge of the dynamics of a staff team if he or she is to have a leadership role and be able to promote understanding in the rest of the team. If the consultant's training has been insufficient, it will be hard for them to support other members of the team to develop their own capacities in this direction. Poor training in human relations skills of mental health nurses and other team members undermines their capacity to manage disturbed patients and cope with their own emotional responses to the work. This underfunctioning leads to the high staff turnover and stress-related illness which is currently evident in many mental health teams.

Community mental health work requires the psychiatrist to have special skills and knowledge of group and projective processes to encourage the team to work effectively and to facilitate their capacity to cope with the strain and pressure from disturbed patients. The success of the team's work requires the staff group to communicate well and support each other appropriately. A well-functioning group of staff will then provide a safe and contained space in which the patient's difficulties and negative attitudes can be understood. Therapeutic interventions can then be introduced at a level that is acceptable and relevant to the individual patient or family.

\section{Group functioning}

The understanding of group functioning in a ward or community hostel will be vital to managing the interaction between members of the staff team. Psychotherapeutic models can provide important insight into group processes, where primitive states of mind can come to dominate both the patient and the staff interactions (Bion, 1961). There has often been confusion in this area because of a failure to distinguish between groups which aim to provide for the personal psychological needs of the members and staff groups which aim to help the members of the therapeutic team to understand more fully their own functioning and their interaction with patients as a staff team. This second type of group is likely to be effective in improving the team's efficiency. Groups aimed at the staff's own psychological needs should be provided outside the setting of the clinical team. Attempts to provide for both personal psychological support and team functioning in the same group are likely to prove confusing.

Where a psychiatric team finds itself involved in a deteriorating and failing capacity to contain patients, the staff need to know how to recruit appropriate help in order to understand what is happening. The problems of containment will be numerous in in-patient settings, where some patients will project their disturbances into the staff group, leading to serious disruption of a safe clinical environment. This is an aspect of the management of an institution which overlaps extensively with clinical work and requires a combined knowledge of the dynamics of individual interactions and of the institution. As one of the leaders of the system, the consultant psychiatrist needs a thorough grasp of these dynamics. He will often need to take action to remedy the problems in a malfunctioning ward, or provide input to prevent emotionally-damaging situations developing.

\section{Staff stress and 'burn-out': can psychotherapy techniques help?}

The increasing problems of staff stress and morale in the mental health service - with high turnover and many trained staff abandoning the field - have also applied to psychiatrists, with problems of recruitment and an increase in early retirement leading to a shortage of consultants and SHOs. 
The inevitable stress of providing a caring service for disturbed patients in the mental health service deserves better recognition (Obholzer, 1997). In a small number of mental health units, staff support groups are provided, but this may be of limited value if staff do not have appropriate training. On the whole, training in psychotherapeutic methods is likely to enhance the consultant's personal capacity to cope, and also enables them to seek appropriate support for themselves and the team, and ensure that the team has access to appropriate training.

During their early career, many psychiatrists have been given poor training and support for the personal aspects of their work with difficult patients. As a result, some may have got into difficulties, to the extent that they have felt emotionally hurt. As a consequence, they have protected themselves emotionally by emphasising the more concrete medical management of aspects of their practice. SHOs and registrars sometimes feel unable to cope with an emotional situation with a patient because they have not had appropriate support and training for work, which has an impact on their own emotions. Work discussion groups can be an important source of learning, to avoid this problem and provide mutual support for trainees. Individual psychotherapy supervision can provide understanding of what has become so difficult and this may become the basis of an important learning experience for the trainee. Supervision as part of training is essential to prevent the development of defensive forms of practice which are detrimental to the effective and sensitive work of the clinician and the team.

It is necessary to distinguish psychotherapeutic supervision - which examines interaction with the patient, including the doctor's emotional responses - from the day-to-day management supervision, which directs the trainee in the general management of the patient's psychiatric care. Psychotherapeutic supervision will have a significant impact on staff stress, because it enables the mental health worker to make more sense of difficulties with the patient, and so able to deal with them with less personal stress.

\section{Dynamic psychiatry}

Dynamic psychiatry is built on the understanding of psychotherapeutic principles and provides an important set of ideas in considering the aims of training in psychiatry (Cawley, 1973). This approach to psychiatry is based on the use of psychodynamic concepts to throw light on the formation of symptoms, the setting up of defences, the interaction between the patient and staff and the effect of unconscious processes in the individual's psychiatric symptomatology. Dynamic psychiatry recognises that personality and childhood psychological development will play an influential part in determining adult disturbance. An understanding of developmental processes will be important in recognising the conflicts faced by an individual in the different stages of the life cycle.

Dynamic psychiatry attempts to understand how powerfully the inner world - with its roots in the past - and the outer world of the present day interact to affect the individual's personality and their symptoms. The repetition of the patterns of childhood relationships in adult life is an important aspect of this.

Dynamic psychiatry adds another dimension to psychopharmacology and the use of behavioural and cognitive approaches. This allows a threedimensional approach to psychiatric practice, integrating the basic principles of psychotherapy into the practice of psychiatry to improve its range and depth. The argument in favour of the training

Box 3. Key contributions of psychotherapy training to the consultant psychiatrist's role

Improves interviewing skills based on understanding the patient-doctor and doctor-patient interaction

Deepens the clinician's understanding of the psychological aspects of the patient's personality and symptomatology

Enhances the leadership role of the consultant psychiatrist in understanding group processes and projective mechanisms in the mental health team and in the in-patient unit or community hostel

Can promote a better understanding of managing dependency and supportive counselling, in which the patient's needs for support have to be balanced with more problematic aspects of dependency

The application of therapeutic community techniques adapted to the management of in-patient units

Understanding the causes of staff stress and burn-out and the need for appropriate staff support, including groups 
of consultant psychiatrists in psychotherapy is the desire to increase their capacity to practise dynamic psychiatry. This is much more important than their ability to practise psychotherapy itself as a technique of treatment. An understanding of dynamic psychiatry enhances the psychological aspects of the psychiatrist's work and encourages the development of a humane and thoughtful psychiatric practice with a sophisticated view of the doctor's interaction with the patient. Patients increasingly expect that the psychiatrist should be a personal doctor as a part of an up-to-date mental health service, and this is the basis of psychiatric practice in many advanced societies.

\section{Conclusion}

In this paper, I have reviewed the arguments which support the present position of the College - that

Box 4. Dynamic psychiatry: a third dimension to psychiatric practice

Complementary to psychopharmacology and to cognitive-behavioural methods

Brings the dimension of unconscious processes and developmental history into understanding the patient's problems

Links the origins of symptoms to dynamic interaction between the patient's inner world and significant figures in the patient's life and important life events

Introduces a new dimension in managing difficult interactions, particularly negative responses to treatment

Allows a fuller understanding of psychic defences, e.g. gain from illness role or resistance to treatment

Adopts a complex view of the patient-doctor interaction, including unconscious elements. Deepens the psychiatrist's understanding of his or her personal reactions to the patient

Allows an understanding of the staff's emotional reactions to patients' projections psychotherapy training is a vital part of general professional psychiatric training and therefore is mandatory as part of each general professional training scheme.

The conclusion I draw is that psychotherapy training enhances many important aspects of the role of the consultant psychiatrist, especially where the role requires the leadership of a multi-disciplinary team, demanding a high level of skill in understanding the team's functioning. Psychotherapy skills are an important part of the clinical skills and practice of consultant psychiatrists in their day-to-day work with patients, and in their capacity to cope emotionally with the work. In addition to their own personal clinical skills, they need to be able to assess patients for their suitability for a range of psychotherapeutic approaches.

The integration of psychotherapeutic practice into psychiatry within dynamic psychiatry will help to meet the needs of a population with an increased expectation of high standards of care from the mental health service.

\section{References}

Balint M. (1957). The doctor, his patient and the illness. London: Pitman Medical.

Balint M. (1968). The Basic Fault. Therapeutic Aspects of Regression. London: Tavistock Publications.

Balint M. \& Balint E. (1961). 'The doctor's emotions'. Chapter 6 in Psychotherapeutic Techniques in Medicine. London: Tavistock Publications.

Bion W. (1961) Experiences in groups and other papers. London: Tavistock Publications.

Cawley R. (1973). 'The teaching of psychotherapy' in Association of University Teachers of Psychiatry Newsletter, January, 19-36.

Dobson F. (1998) Third Way for Mental Health. 98/311. London: Department of Health.

Feldman M. The dynamics of reassurance. International Journal of Psycho-anal. 1993, 74, 275-284.

Gibb E. \& Temple N. (1998). Unpublished report to Court of Electors.

NHS Executive (1996). NHS Psychotherapy Services in England: Review of Strategic Policy. London: Department of Health.

NHS Executive (1998). A First Class Service: Quality in the New NHS. HSC 1998/113. Department of Health.

Norton K. \& Hinshelwood R. (1996) Severe personality disorder: treatment issues and selection for in-patient psychotherapy. British Journal of Psychiatry, 168, 723-731.

Obholzer A. (1997) 'Challenge, Change and Sabotage'. Paper given at Queen Mary and Westfield College Public Policy Seminar on 'Developing Policy and Practice on Mental Health'.

Royal College of Psychiatrists (1993) Guidelines for Psychotherapy Training as Part of General Professional Psychiatric Training. Council Report CR27. London.

Stewart H. (1989) Technique at the Basic Fault / Regression. Reprinted in International Journal of Psycho-anal, 1989, 70, 221-230.

Temple N. et al (1997) Interpretative group psychotherapy and dependent day hospital patients, a preliminary investigation. International Journal of Social Psychiatry, 43, 2, 116-128. 


\section{Multiple choice questions}

1. Type A psychotherapy:

a is only practised by specialist psychotherapists

b can be an integrated part of CPA

c is only suitable for neurotic disorders

d is not appropriate for primary care practice

e should be an approach used by all mental health professionals.

2. Dynamic psychiatry:

a is only applicable to neurotic patients

b can make a contribution to understanding patients' resistance to treatment

c is contra-indicated where psychopharmacological treatment is used

d may be of help in understanding the origin of a patient's symptoms in their history

e is only applicable with well-motivated and cooperative patients.
3. Psychotherapy training:

a equips the consultant psychiatrist to do a limited amount of psychological treatment but has no contribution to make to overall competency as a psychiatrist

b may enhance a consultant psychiatrist's ability to manage an in-patient unit effectively

c is not relevant to supportive counselling

d may have a role in dealing with staff stress and burn-out

e makes no contribution to the management of dependent patients.

\begin{tabular}{|lll|}
\hline MCQ answers & & \\
\hline 1 & 2 & 3 \\
a F & a F & a F \\
b T & b T & b T \\
c F & c F & c F \\
d F & d T & d T \\
e T & e F & e F \\
\hline
\end{tabular}

\section{New from Gaskell}

\section{Mental Health of Ethnic Minorities: an annotated bibliography}

\section{Edited by Dinesh Bhugra}

This book will be an invaluable and useful source for mental health professionals who have dealings with minority ethnic groups in their clinical and research practice. It provides an annotated bibliography of recent important papers which describe research on mental health of minority ethnic groups in the UK. Collected from a number of resources these papers highlight the current status of the research and will also be useful for researcher and practitioner alike.

Clinicians and researchers will find this book useful in informing their clinical practice and help formulate research ideas. This volume will be of use to mental health professionals as well as those who are interested in the field of cross-cultural psychiatry.

July 1999, £10.00, Paperback, ISBN 1901242315 